\title{
Influence of relative density on the cyclic shear strength of sands
}

\section{Influence de la densité relative sur la résistance au cisaillement cyclique des sables}

\author{
ARAB A. ${ }^{1}$, Marwan Sadek ${ }^{2,3}$ and SHAHROUR I. ${ }^{2}$ \\ ${ }^{1}$ Laboratory of Materials sciences and Environment \\ Civil Engineering Department, University Hassiba Ben Bouali, 02000 Chlef, Algeria, \\ ${ }^{2}$ Polytech-Lille, France, \\ ${ }^{3}$ Lebanese University, Lebanon.
}

\begin{abstract}
Abstrtact. This paper presents a laboratory study of the influence of relative density on the liquefaction potential of a soil. The study is based on undrained triaxial tests that were performed on samples with relative density Id = $0.15,0.5$ and 0.65 . The article is composed of three parts. First, we present the materials and characteristics of the studied sands. the second part deals with the procedure and the device used. The third part studies the influence of the relative density on the liquefaction potential of the three sands (Hostun Rf, Chlef and Rass). This study also makes it possible to explore the influence of granulometry on the liquefaction potential. The results of the tests show that concordant results have been obtained which clearly show that the increase of the relative density leads to a significant improvement in the resistance to liquefaction of the sands. This effect is very significant when the initial relative density $\mathrm{Id}=0.50$ to $\mathrm{Id}=0.65$.
\end{abstract}

\begin{abstract}
Résumé: Cet article présente une étude en laboratoire de l'influence de la densité relative sur le potentiel de liquéfaction d'un sol. L'étude est basée sur des essais triaxiaux non drainés qui ont été effectués sur des échantillons ayant une densité relative $\mathrm{Id}=0,15,0,5$ et 0,65 . L'article est composé de trois parties. Dans un premier temps nous présentons les matériaux et les caractéristiques des sables étudiés. la seconde partie traite le mode opératoire et le dispositif utilisé. La troisième partie étudie l'influence de la densité relative sur le potentiel de liquéfaction des trois sables (Hostun Rf, Chlef et Rass). Cette étude permet également d'explorer l'influence de la granulométrie sur le potentiel de liquéfaction. Les résultats des essais montrent que des résultats concordants ont été obtenus et qui montrent d'une manière très claire que l'augmentation de la densité relative conduit à une importante amélioration de la résistance à la liquéfaction des sables. Cet effet est très significatif lorsque la densité relative initiale $\mathrm{Id}=0,50$ à $\mathrm{Id}=0,65$.
\end{abstract}

\section{Introduction}

La résistance à la liquéfaction d'un site peut-être améliorée si celui-ci est sujet à un haut risque de liquéfaction, en agissant sur l'un de ces paramètres influents sur la résistance. Actuellement les méthodes les plus utilisées pour la stabilisation des sites agissent sur la densité relative et les conditions de drainage du sol. Récemment de nouvelles méthodes de stabilisation des sites liquéfiables ont été envisagées. Ces dernières consistent à améliorer la résistance à la liquéfaction par inclusion de nappes de géotextile, par augmentation de la perméabilité du sol.

La densité relative affecte d'une manière très sensible le potentiel de liquéfaction des sols. La figure 1 donne les résultats obtenus par Tatsuoka et al. (1986b) sur le sable de Toyoura. On constate que la résistance à la liquéfaction augmente de façon linéaire avec la densité relative jusqu'à une valeur de densité relative $\operatorname{Dr}=0,70$. Après cette valeur, on remarque une augmentation importante de la résistance avec l'augmentation de la densité relative.

Polito et Martin (2001), ont réalisé une série d'essais triaxiaux sur des échantillons de sable (Monterrey et Yatesville) mélangé avec des fines non plastiques. Ils constatent une tendance linéaire entre l'augmentation de la densité relative et la résistance à la liquéfaction pour les échantillons de sol ayant une teneur en fines en dessous du seuil limite (seuil limite des fines étant le passage d'une matrice de sol à prédominance sable à matrice contrôlée par les fines), cependant la résistance à la liquéfaction au-dessus du seuil limite est aussi contrôlée par la densité relative de l'échantillon, cependant la résistance des sols est nettement inférieure par rapport aux 
même sols qui se trouvent en dessous du seuil avec la même densité relative.

Dans cet article, on présente une étude au laboratoire sur l'influence de la densité relative sur la résistance à la liquéfaction. Ces essais permettent de mieux comprendre l'influence de la densité relative sur le comportement cyclique des sables. L'article est composé de trois parties; dans la première on présente les matériaux utilisés ; la seconde partie le mode opératoire et le dispositif utilisé ; la dernière donne une analyse des résultats expérimentaux des essais réalisés et discute de l'influence de l'influence de la densité relative sur le comportement cyclique des sables.

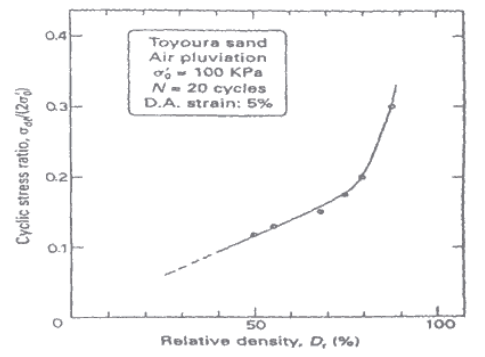

Fig. 1. Effet de la densité relative sur la résistance à la liquéfaction(Tatsuoka et al.,1986b)

\section{Matériaux et dispositif expérimental.}

\subsection{Matériaux}

Trois matériaux ont été utilisés dans cette étude à savoir le sable de Chlef et Rass (Algérie) et le sable d'Hostun Rf (France). Le sable de Chlef comme son nom l'indique provient du lit de l'Oued-Chlef qui traverse la ville de Chlef. Le sable d'Oued Rass provient des abords du lit d'Oued Rass (confluent Oued Chlef et Oued Rass). Le sable d'Hostun Rf provient des usines SIKA implantées à 26730 Hostun (Drôme) sur le flanc ouest du Vercors, la carrière d'où provient le sable est implantée dans une série épaisse de sables d'origines Eocène remplissant des poches karstiques. Le sable de Chlef est un sable moyen ayant une forme arrondie (sable alluvionnaire), avec un diamètre moyen $\mathrm{D}_{50}=0,61 \mathrm{~mm}$. Le sable d'Oued Rass est aussi un sable moyen de forme arrondie (sable alluvionnaire) avec un diamètre moyen $\mathrm{D}_{50}=0.39 \mathrm{~mm}$. Le sable d'Hostun est considéré comme un matériau de référence dans de nombreux laboratoires en France. De nombreux essais ont été réalisés sur ce sable (Colliat 1986, Fargeix 1986, Flavigny et al. 1990, Biarez et Ziani 1991, Lancelot et al. 1996, Doanh et al. 1997, Al mahmoud 1997, Hoque et Tatsuoka 1998). Il s'agit un sable moyen ayant une forme angulaire avec un diamètre moyen $\mathrm{D}_{50}=0,471$. La figure 2 montre les courbes granulométriques des matériaux utilisés. Ces courbes ont été obtenues par tamisage sur une série complète de tamis. Le tableau 1 donne les caractéristiques de ces sables.

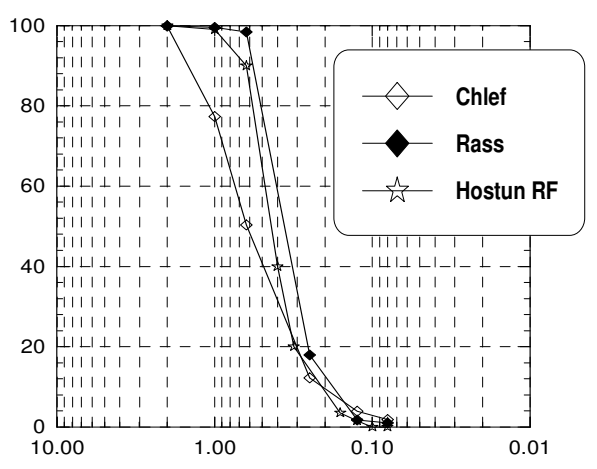

Fig. 2.Courbegranulométrique

Use A4 paper size $(210 \times 297 \mathrm{~mm})$ and adjust the margins to those shown in Table 1 . The final printed area will be $172 \times 252 \mathrm{~mm}$.

Tableau 1. Caractéristiques des sables utilisés

\begin{tabular}{|c|c|c|c|}
\cline { 2 - 4 } \multicolumn{1}{c|}{} & \multicolumn{3}{c|}{ Sable } \\
\hline Propriétés & Chlef & Rass & Hostun \\
\hline $\begin{array}{c}\text { Masse spécifique } \\
\square_{\mathbf{s}}\left(\mathrm{g} / \mathrm{cm}^{3)}\right.\end{array}$ & 2,68 & 2,664 & 2,654 \\
\hline $\mathrm{e}_{\max }$ & 0,854 & 0,770 & 0,983 \\
\hline $\mathrm{e}_{\min }$ & 0,535 & 0,490 & 0,622 \\
\hline $\begin{array}{c}\mathrm{Cu} \\
\left(\mathrm{D}_{60} / \mathrm{D}_{10)}\right.\end{array}$ & 3,38 & 2,42 & 2,27 \\
\hline $\mathrm{D}_{10}$ & 0,225 & 0,227 & 0,19 \\
\hline $\mathrm{D}_{50}$ & 0,61 & 0,39 & 0,471 \\
\hline $\begin{array}{c}\text { Forme des } \\
\text { particules }\end{array}$ & Arrondi & Arrondie & Angulai \\
\hline & $\mathrm{e}$ & & re \\
\hline
\end{tabular}

\subsection{Dispositif expérimental}

Le dispositif expérimental utilisé est présenté schématiquement dans la figure 3 . Il comprend :

- Une cellule triaxiale autonome type Bishop et Wesley (Bishop et Weslay, 1975),

- Trois contrôleurs de pression/volume type GDS (200cc),

- Une pompe à vide relié à un réservoir afin de désaérer l'eau déminéralisée,

- Un micro-ordinateur équipé d'un logiciel permettant le pilotage de l'essai et l'acquisition des mesures. 


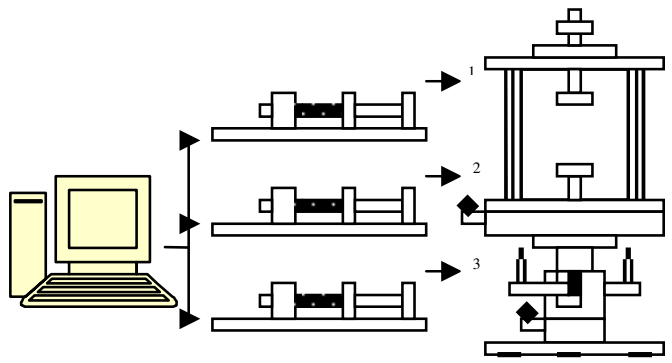

(1) application de la pression dans la cellule

(2) contrôle de la pression interstitielle ou variation de volume de l'échantillon

(3) contrôle du déviateur

Fig. 3. Dispositif expérimental utilisé

Elle est constituée de deux chambres. La chambre supérieure contient l'échantillon et les deux embases servant à appliquer la charge axiale sur celui-ci. La pression maximale que peut supporter cette chambre est de $2 \mathrm{MPa}$. La partie inférieure consiste en une presse hydraulique. Elle est constituée d'une chambre métallique dans laquelle un piston peut coulisser. L'extrémité du piston est reliée à l'embase inférieure qui applique la charge axiale à l'échantillon. Comme la section du piston est connue, la force et le déplacement axial appliqué par le piston sont déduits directement de la pression et de la variation de volume dans la chambre. Ces dernières sont contrôlées par un GDS qui permet d'appliquer le chargement axial en contrainte ou en déformation contrôlée. L'échantillon est balayé tout d'abord par du gaz carbonique $\mathrm{CO}_{2}$ pendant 20 minutes ensuite on fait passer l'eau désaérée et déminéralisée à travers l'échantillon pour le saturer. Les échantillons sont consolidés isotropiquement pour atteindre la valeur de contrainte de confinement effective précédent le chargement. La contre pression utilisée pour nos essais est de $400 \mathrm{kPa}$, la contrainte de confinement est égale à $500 \mathrm{kPa}$. Le degré de saturation des échantillons estévalué en mesurant le coefficient de Skempton $B$ après consolidation $(\mathrm{B}=\Delta \mu / \Delta \sigma)$.

\section{Résultats des essais}

\subsection{Essais cycliques sur le Sable d'Hostun}

Les résultats de l'ensemble des essais réalisés sont résumés sur les figures 4.a et 4.b. La première illustre l'influence de la densité relative sur le potentiel de liquéfaction. Elle montre d'une manière très claire que l'augmentation de la densité relative conduit à une translation importante des courbes de potentiel de liquéfaction vers la droite. C'est-à-dire, que l'augmentation d la densité relative améliore la résistance à la liquéfaction de ce sable. Ce résultat est cohérent avec l'influence de la densité relative sur l'augmentation de la dilatance du sable.

La figure 5.1b montre l'influence de la densité relative sur la résistance à la liquéfaction qui a définie par l'amplitude du chargement induisant la liquéfaction après 15 cycles.
Cette figure montre bien que la résistance à la liquéfaction augmente avec l'augmentation de la densité relative et la diminution de l'amplitude du chargement.
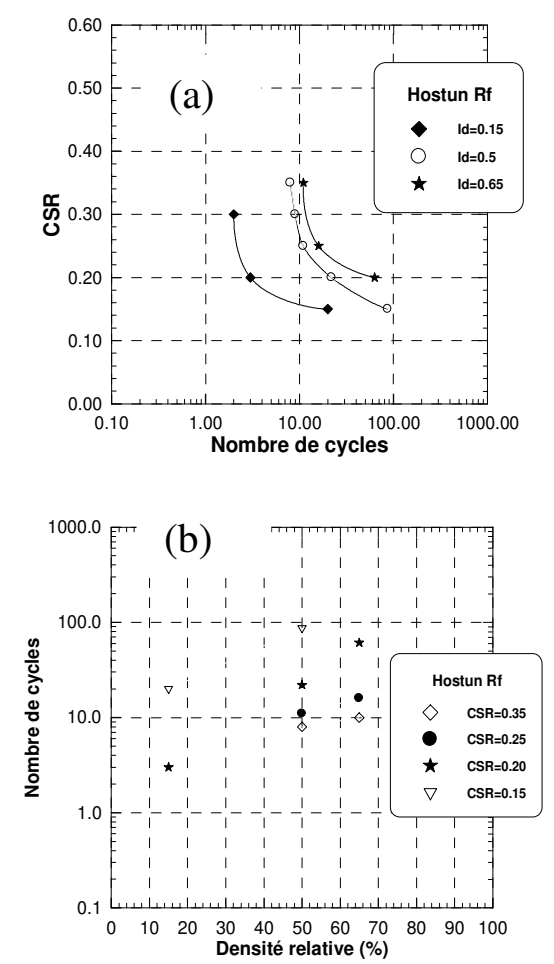

Fig.4. Influence de la densité initiale sur la liquéfactiondu sable d'Hostun Rf

(a) Sur le potentiel de liquéfaction,

(b) sur la résistance à la liquéfaction,

\subsection{Essais cycliques sur le sable de Rass}

Les résultats de l'ensemble de ces essais réalisés sur les échantillons du sable d'Oued Rass sont illustrés sur la figure 5. La figure 5.a illustre l'influence de la densité relative sur le potentiel de liquéfaction. Elle montre d'une manière très claire que l'augmentation de la densité relative conduit à une importante translation importante de la courbe de potentiel de liquéfaction vers la droite. C'est-à-dire, que l'augmentation de la densité relative améliore la résistance à la liquéfaction de ce sable.

La figure 5.b montre l'influence de la densité relative sur la résistance à la liquéfaction définie par l'amplitude du chargement induisant la liquéfaction après 15 cycles. Cette figure montre bien que la résistance à la liquéfaction augmente avec l'augmentation de la densité relative et la diminution de l'amplitude du chargement. La différence entre la résistance du sable à une densité relative $\mathrm{Id}=50$ et celle pour $\mathrm{Id}=0,65$ est très importante en comparaison avec la différence observée sur le sable d'Hostun. 

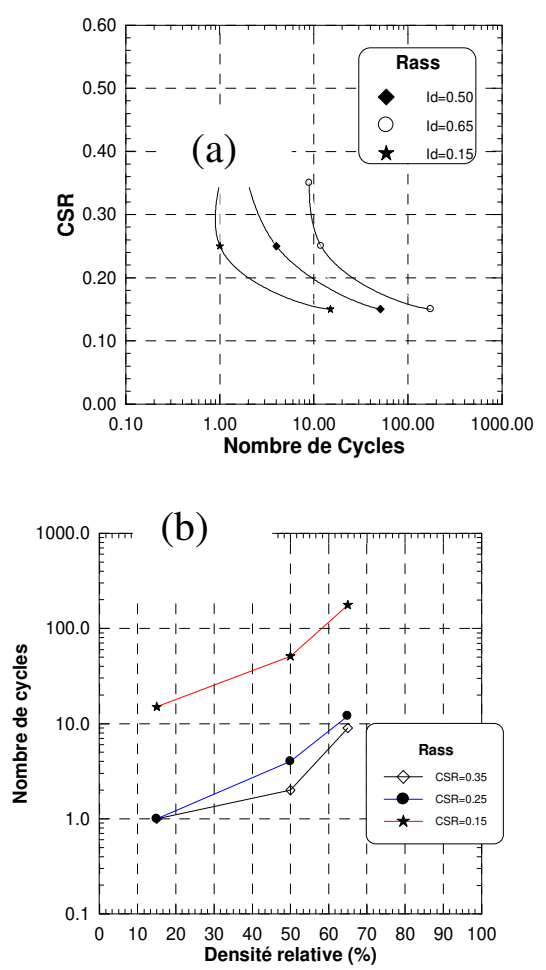

Fig.5. Influence de la densité initiale sur la liquéfactiondu sable de Rass.

(a) Sur le potentiel de liquéfaction,

(b) Sur la résistance à la liquéfaction,

\subsection{Essais cycliques sur le sable de Chlef}

La figure 6 illustre les résultats des essais cycliques sur le sable de Chlef qui a subi une liquéfaction lors du séisme de 1980. La figure 6.a illustre l'influence de la densité relative sur le potentiel de liquéfaction. Elle montre clairement que l'augmentation de la densité relative conduit à une augmentation de la résistance à la liquéfaction de ce sable. La figure 6.b montre l'influence de la densité relative sur la résistance à la liquéfaction définie par l'amplitude du chargement induisant la liquéfaction après 15 cycles. Cette figure montre bien que la résistance à la liquéfaction augmente avec l'augmentation de la densité relative et la diminution de l'amplitude du chargement. La différence entre la résistance du sable à une densité relative $\mathrm{Id}=50$ et celle pour Id $=0,65$ est très importante; elle ressemble à celle observée sur le sable de Rass (Figure 5.b).

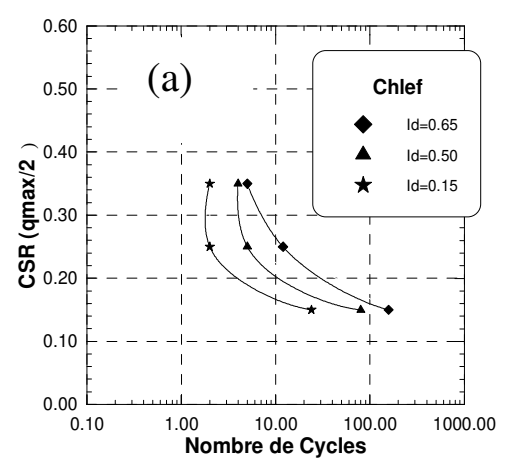

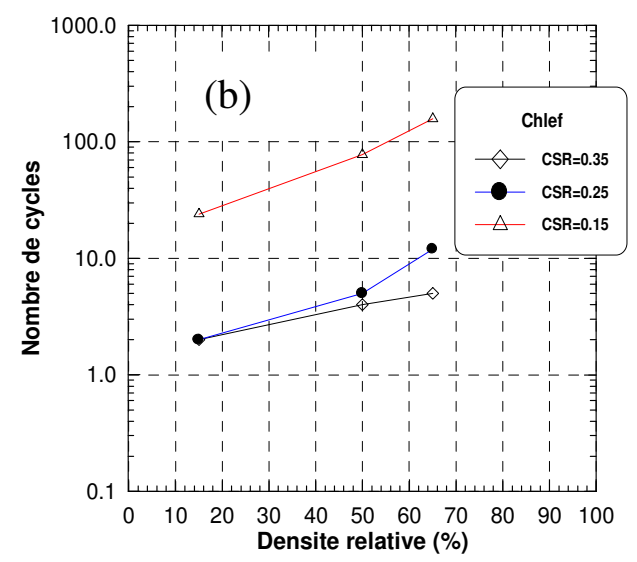

Fig.6. Influence de la densité initiale sur la liquéfaction du sable de Chlef.

(a) Sur le potentiel de liquéfaction,

(b) Sur la résistance à la liquéfaction,

La courbe 7 illustre l'évolution de la résistance à la liquéfaction en fonction de la densité relative (Dr). On déduit à partir de cette figure que le sable d'HostunRf dont les grains ont une forme angulaire présente une résistance à la liquéfaction plus grande que ceux des sables de Rass et Chlef qui sont des sables alluvionnaires et de forme arrondie. La résistance à la liquéfaction augmente linéairement avec l'augmentation de la densité relative.

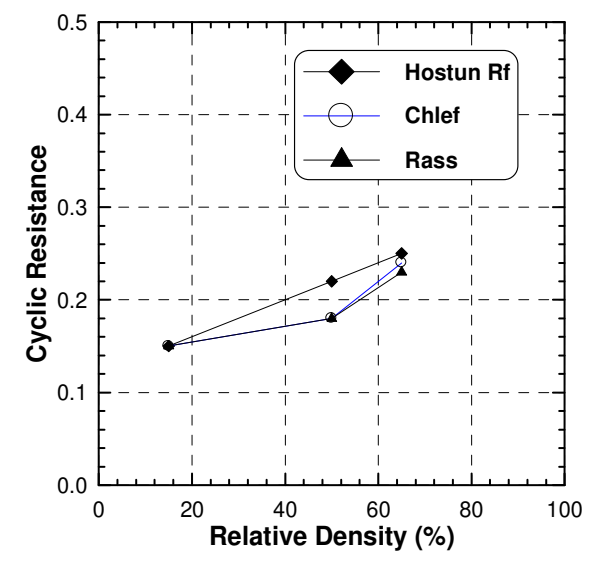

Fig.7. Evolution de la résistance en fonction de la densité relative.

\section{Conclusions}

Ce travail a été réalisé dans le but de mettre en évidence l'influence de la densité relative sur le potentiel de liquéfaction de trois sables à savoir le sable d'Hostun Rf (France) et les sable de Rass et Chlef (Algérie). Les essais ont été réalisés sur des échantillons lâches, moyennement denses et denses avec des degrés de saturations caractérisés par le coefficient de Skempton B dépassant les $95 \%$.

Les résultats des essais cycliques sur le sable d'Hostun RF dont les grains ont une forme angulaire montre que l'augmentation de la densité relative mène à 
une augmentation de la dilatance du sable et par conséquent la résistance à la liquéfaction augmente.

Les résultats des essais cycliques sur le sable de Rass dont les grains ont une forme arrondie montrent bien que la résistance à la liquéfaction augmente avec l'augmentation de la densité relative et la diminution de l'amplitude du chargement.

Les résultats des essais cycliques sur la sable de Chlef aussi les grains ont une forme arrondie et peu grossier par rapport au sable de Rass ont montré que la densité relative influe d'une manière significative sur la résistance à la liquéfaction, elle augmente avec l'augmentation de la densité. La différence entre la résistance du sable à une densité relative $\mathrm{Id}=50$ et celle pour Id $=0,65$ est très importante ; elle ressemble à celle observée sur le sable de Rass.

La résistance à la liquéfaction du d'Hostun $\mathrm{Rf}$ est supérieure par rapport aux autres sables (Chlef et Rass) et qui peut être expliquer par le faite que le sable d'Hsotun Rf est de caractère dilatant que les autres sables de Chlef et Rass qui sont des sables à caractère contractant.

\section{References}

1. Al Mahmoud M. « Etude en laboratoire du comportement des sables sous faibles contraintes», Thèse de doctorat en génie civil à l'USTL, Lille (1997)

2. Biarez J. \&Ziani $\mathrm{F}$ « Introduction aux lois de comportement des sables très peu denses», Revue Française de Géotechnique, Vol. 54, pp. 65-73(1991)

3. Bishop A. W. \& Wesley L.D. «A hydraulic triaxial apparatus for controlled stress path testing», Geotechnique, Vil.25, N 4, pp. 657-670 (1975)

4. Colliat J.L.: « Comportement des matériaux granulaires sous forte contraintes, influence de la nature minéralogique du matériau étudié», Thèse de doctorat de spécialité, IMG, Grenoble (1986)

5. Doanh, T., Ibraim, E., et Matiotti, R.(1997): " Undrained instability of very loose Hostun sand in triaxial compression and extension.Part 1 : experimental observations.Mechanics of CohesiveFrictional Materials, 2 :47-70 (1997)

6. Flavigny E., Desrues J.\&Palayer B. « Le sable d'Hostun Rf», Revue Française de Géotechnique, N53, pp.67-70 (1990)

7. Gargeix D. «Conception et réalisation d'une presse triaxiale dynamique-application à la mesure des propriétés des sols sous sollicitations sismiques», Thèse de doctorat, IRIGM, Grenoble(1986)

8. Lancelot L., Shahrour I. \& Al Mahmoud M. « Comportement du sable d'Hostun sous faibles contraintes», Revue Française de Geotechnique, N74, Janvier 1996, pp.63-74 (1996)

9. Polito C.P. \& Martin J.R. « Effects of nonplastic fines on the liquefaction resistance of sands», Journal of Geotechnical and Geoenvironmental Engineering, Vol., 127, N5, pp. 408-415(2001)

10. Tatsuoka F., Miura S., Yoshimi Y., Yasuda S., \&Makihara Y. «Cyclic undrained triaxial strength of sand by a cooperative test program, Soils and foundations», 26, 117-128(1986b)

11. Hoque E. \&Tatsuoka F. « Anisotropy in the elastic deformation of granular materials », Soils and Foudations, Vol.38, N 1, pp. 163-179(1998) 